\title{
Calidad de vida en familias de personas con discapacidad
}

Quality of life in families of persons with disability

Qualidade de vida em famílias de pessoas com deficiência

\section{Miguel Ángel Verdugo Alonso ${ }^{1}$ \\ Leonor Córdoba Andrade ${ }^{2}$ Alba Rodríguez Aguilella ${ }^{3}$}

Recibido: 19 de noviembre de 2019

Aprobado: 31 de enero de 2020

Publicado: 2 de marzo de 2020

Cómo citar este artículo:

Verdugo Alonso, M. Á., Córdoba Andrade, L. y Rodríguez Aguilella, A. (2020). Calidad de vida en familias de personas con discapacidad. Pensando Psicología, 16(1), 1-23. doi: https://doi.org/10.16925/2382-3984.2020.01.01

Artículo de investigación. https://doi.org/10.16925/2382-3984.2020.01.01

1 Universidad de Salamanca

ORCID: https://orcid.org/0000-0002-5802-8220

2 Universidad del Tolima

ORCID: https://orcid.org/0000-0003-2006-1639

3 Servicio de Orientación Educativa, Psicopedagógica y Profesional de la Comunidad Valenciana

ORCID: https://orcid.org/0000-0002-9026-7360 


\section{Resumen}

Objetivo: establecer los avances en la comprensión de la calidad de vida en familias de personas con discapacidad en el marco de la atención centrada en la familia.

Metodología: a partir de las relaciones entre familia y discapacidad desde el modelo de calidad de vida, se analizan los principales estudios en España y América Latina.

Resultados: se evidencia que la investigación en calidad de vida familiar ha mostrado progresos importantes en el desarrollo de prácticas profesionales y organizacionales orientadas al fortalecimiento de la autonomía de las familias para la toma de decisiones frente a aspectos cruciales de sus vidas y; un marcado interés en los profesionales y en las organizaciones por contar con herramientas de calidad de vida familiar que les permitan evaluar el impacto de su práctica y sus servicios.

Conclusión: la mejora en la calidad de vida familiar resulta fundamental en el desarrollo personal y la inclusión social de las personas con discapacidad.

Palabras clave: familias, discapacidad, calidad de vida, calidad de vida familiar, evaluación.

\section{Abstract}

Aim: To establish advances in the understanding of the quality of life in families of people with disabilities within the framework of family-centered attention.

Methodology: From the relationships between family and disability from the model of quality of life, the main studies in Spain and Latin America are analyzed.

Results: it is shown that, research on family quality of life has shown significant progress in the development of professional and organizational practices aimed at strengthening the autonomy of families for decision-making in the face of crucial aspects of their lives and; a strong interest in professionals and organizations to have quality tools of family life that assesses the impact of their practice and their services.

Conclusion: The improvement in the quality of family life is fundamental in the personal development and social inclusion of people with disabilities.

Keywords: families, disability, quality of life, family quality of life, assessment

\section{Resumo}

Objetivo: Estabelecer avanços na compreensão da qualidade de vida em famílias de pessoas com deficiência no âmbito da atenção centrada na família.

Metodologia: A partir das relações entre família e deficiência desde o modelo de qualidade de vida, analisam-se os principais estudos na Espanha e na América Latina.

Resultados: evidencia-se que, as pesquisas sobre qualidade de vida familiar têm mostrado avanços significativos no desenvolvimento de práticas profissionais e organizacionais voltadas ao fortalecimento da autonomia das famílias para a tomada de decisões frente a aspectos cruciais de suas vidas e; um forte interesse de profissionais e organizações por ferramentas de qualidade de vida familiar que avaliem o impacto de sua prática e seus serviços.

Conclusão: A melhoria da qualidade de vida familiar é fundamental para o desenvolvimento pessoal e inclusão social das pessoas com deficiência.

Palavras-chave: famílias, deficiência, qualidade de vida, qualidade de vida familiar, avaliação 


\section{Introducción}

La familia siempre ha desempeñado un rol de suma relevancia para mantener o alcanzar condiciones de satisfacción y bienestar en la vida de las personas que tienen algún tipo de discapacidad. Prueba de esto es el hecho de que personas con discapacidad (en adelante PcD), que tienen un alto nivel de satisfacción o éxito profesional, se relacionan de manera muy estrecha con familias que les brindan apoyo y favorecen su progreso individual. En estas familias, se destaca el esfuerzo y la dedicación exclusiva asumida por la madre (Cuevas, Salcedo y Díaz, 2019; Kovac y Memisevi, 2017). Por el contrario, cuando la familia no responde a los intereses y el desarrollo personal de uno de sus integrantes con discapacidad, la repercusión sobre la persona es negativa, lo que se evidencia en prácticas de sobreprotección, dependencia y poco fomento de la autodeterminación y la inclusión en diferentes contextos lo que termina limitando su desarrollo pleno (Aya y Córdoba, 2013; Muñoz, 2019).

Así, la familia no es solamente importante en el ámbito privado, sino que su actividad también incide directa e indirectamente en lo público, en relación con diversos aspectos del entorno social y comunitario que rodea a la PcD. De hecho, se ha comprobado que el desarrollo de estrategias de atención y provisión de apoyos por parte de las organizaciones y asociaciones que apoyan a las PcD, favorece la consolidación del liderazgo iniciado por sus familiares, que se constituye en una herramienta para medir dicha provisión de apoyos (Kovac y Memisevic, 2017), generalmente, brindados por profesionales comprometidos, con quienes se establecen acuerdos de cooperación (Córdoba y Verdugo, 2015), con un propósito común: favorecer avances en la inclusión social de la PcD, así como mejorar su autonomía y los resultados personales.

No obstante, solamente hasta finales del siglo pasado, hubo un giro significativo en el foco de interés de las prácticas de atención a las PcD, en las que se comenzó a hablar de las familias y las necesidades e interacciones que presentaban cada uno de sus integrantes con la familia extensa y con su comunidad inmediata (Martínez, Fernández, Orcasitas, Montero y Villaescuesa, 2016). De esta manera, se profundizó en el análisis de los roles que han desempeñado los miembros de la familia de una PcD, que van desde ser objeto de intervenciones terapéuticas o formativas, hasta ser considerados como sujetos activos en la determinación de necesidades y la toma de decisiones para favorecer su satisfacción plena (Fernández, Montero, Martínez, Orcasitas y Villaescusa, 2015).

El cambio de visión de la familia y el papel relevante que debe asumir en el mundo de la discapacidad, se acentuó con el nuevo siglo y refleja los avances en la comprensión de la propia discapacidad y los factores o elementos que intervienen en 
ella (Luckasson et al., 2002; Luckasson y Schalock, 2013; Organización Mundial de la Salud, 2001; Schalock et al., 2010). Este cambio se refiere al avance que se produce desde una perspectiva reduccionista de la discapacidad, centrada exclusivamente en los problemas o la "patología", a una concepción social y ecológica, que da más valor a la influencia del ambiente en la situación que tiene la PcD (Schalock y Verdugo, 2007). Así, el bienestar individual, depende de aspectos personales y de determinantes ambientales, siendo estos últimos en los que se puede incidir de manera sostenida a lo largo de su vida.

Para situar adecuadamente las relaciones entre familia y discapacidad, se debe considerar que las personas están influenciadas por múltiples sistemas, desde los micro hasta los macrosistemas (Bronfenbrenner, 1984, 2005; Córdoba, 2017) y, sin lugar a dudas, el microsistema familiar resulta determinante en el desarrollo, el aprendizaje, la independencia, la autonomía y la autodeterminación del individuo. Por lo tanto, sus acciones tendrán un impacto en las necesidades que experimentan cada uno de sus integrantes sobre los aspectos más significativos de su vida, incluyendo a la PcD (Chiu et al., 2013; Rivard et al., 2017), lo que se traduce en calidad de vida.

De ahí que, la calidad de vida (cv) se configura como un marco conceptual pertinente y actualizado, para el desarrollo de prácticas profesionales que garanticen el funcionamiento óptimo de la familia dentro de su hogar y comunidad, dado que este tipo de prácticas soporta el desarrollo integral de sus miembros y, a su vez, contribuye con la estabilidad continua de las sociedades (Schlebusch, Dada y Samuels, 2017). En consecuencia, la cV de la persona va a depender en gran medida de la CV de su familia (Chiu et al., 2013). En este punto radica la importancia de su estudio, como mecanismo para proporcionar objetivos de mejora en el ámbito familiar, los cuales deben contribuir a un mayor grado de bienestar en la PcD (García et al., 2018).

Por eso, con base en este marco de referencia, el propósito de este artículo es mostrar los avances logrados en la comprensión de la CV en familias de PCD en función de la perspectiva de atención centrada en la familia y sus implicaciones. Para ello, se identificarán las relaciones entre familia y discapacidad, desde el modelo de calidad de vida, para luego recoger los resultados de algunos estudios realizados en España y América Latina sobre el tema como insumo para el desarrollo de una práctica profesional y organizacional basada en la evidencia. 


\section{Discapacidad y familia desde el marco de calidad de vida}

Se puede decir que son cuatro los factores que han influido significativamente en el campo de las discapacidades en las últimas tres décadas (Schalock, Verdugo, Gómez y Reinders, 2016). El primero, es el modelo ecológico de la discapacidad que se centra en la interacción persona-medio ambiente y la congruencia entre la competencia personal y las demandas ambientales (Schalock y Luckasson, 2015; Schalock y Verdugo, 2007). El segundo, es el paradigma de apoyos que se basa en la evaluación de las necesidades de estos en las principales áreas de actividad de la vida y la provisión de apoyos individualizados que reducen esta discrepancia entre competencias personales y demandas ambientales (Guillén, Verdugo, Arias y Vicente, 2015; Thompson et al., 2016; Verdugo et al., 2007). El tercer factor, son los principios de la psicología positiva que incluyen la experiencia subjetiva valorada, los rasgos individuales positivos y los valores cívicos (Hart y Sasso, 2011; Wehmeyer, 2013). Y el cuarto, es el reconocimiento internacional de los derechos de las PcD y el potencial de un modelo conceptual de cv individual para operacionalizar los principales artículos contenidos en la Convención de las Naciones Unidas sobre los Derechos de las Personas con Discapacidad (ONU, 2006; Navas et al., 2012; Verdugo et al., 2012).

De ahí la importancia del concepto, el modelo y la teoría de cV (Schalock et al., 2016), pues integra esos cuatro factores descritos en un enfoque basado en valores -equidad, empoderamiento, autodeterminación e inclusión- que se centra en la individualidad de cada persona y que sirve para orientar los cambios que deben darse en la prestación de servicios, en los apoyos y en la evaluación de los resultados personales, organizacionales y sociales (Gómez, Verdugo y Arias, 2010).

Incluso, este constructo ha sido empleado en otro nivel de análisis, como en el caso del estudio de la familia de las PcD, en virtud de los cambios significativos que ha sufrido la definición tradicional de familia, que ha estado ligada exclusivamente a lazos de consanguinidad o vínculos de afinidad legal como el matrimonio (Poston et al, 2003; Turnbull, 2003). Prueba de ello, son los cambios de tipologías o conformación de la familia, que responden a diferentes dinámicas sociales, políticas, económicas, culturales y lingüísticas (Córdoba, González, Obando y Colacouglou, 2013), que implican una reorganización de los roles y funciones que ejercen cada uno de sus integrantes, de modo que estos pueden apoyarse y cuidarse entre sí (Abad, 2016).

Cada familia es similar a las demás en varios aspectos y, a la vez, es única en muchos otros. Se entiende que, si en numerosas ocasiones es complejo responder a las necesidades de una persona, responder a las de una familia puede serlo 
mucho más. Si se tienen en cuenta aspectos como la edad de sus integrantes, su situación laboral o académica, las condiciones del barrio o la población en la que habitan, sus ingresos y gastos, los servicios a que tienen acceso, entre otras variables, la posibilidad de combinaciones es inmensa (García et al., 2018). Si a eso añadimos que los miembros de la familia tienen influencia unos en otros (Abad, 2016), nadie puede dudar de que cada familia sea única y tiene unas necesidades, unos recursos y unas fortalezas que son muy importantes de conocer para poder atenderlos (García et al., 2018).

Aunque la singularidad de cada familia y de cada PcD es evidente, existen también elementos comunes que están presentes en la manera de enfocar los apoyos y las actividades de ayuda necesarias para hacer propuestas eficaces de atención y colaboración. Para ello, es indispensable identificar varios factores subjetivos y objetivos que inciden en la c de estas familias (Giné et al., 2015), que han sido recopiladas con base en referencias teóricas relacionadas con interacción familiar, bienestar emocional, bienestar físico/material, rol parental y apoyo a las PcD (Beach Center, 2001; Hoffman et al., 2006; Turnbull, Summers, Lee y Kyzar, 2007), salud, bienestar financiero, relaciones familiares, apoyo de otras personas, apoyo de servicios relacionados con la discapacidad, influencia de valores, carreras y preparación para carreras, ocio y recreación e interacción comunitaria (Brown, Hong, Shearer, Wang y Wang, 2010; Brown et al., 2006), entre otras.

Desde esta perspectiva, hablar de cV en las familias hoy, supone revisar el impacto que la discapacidad tiene sobre la vida de este microsistema. Y, también, requiere analizar el rol que las familias desempeñan para que la cV de las PcD sea la mejor posible. Se plantea, por tanto, un enfoque centrado en la familia, basado en la cooperación con la familia, que supere el enfoque clínico o terapéutico focalizado en los problemas del sistema familiar (Fernández et al., 2015) y se encamine hacia una visión más inclusiva de ella en cuanto a su capacidad para apreciar y priorizar intereses y adaptarse a los distintos entornos (Turnbull, Turnbull y Kyza, 2009).

El origen del término calidad de vida familiar (CVF) se remonta a finales del siglo pasado, cuando se estableció como un ámbito nuevo de investigación con implicaciones prácticas importantes. Zuna et al. (2009) conceptualizan la CVF como un estado dinámico de bienestar de la familia, que se define de forma colectiva y subjetiva, y que es valorado por sus miembros, en el cual interactúan las necesidades a nivel individual y familiar. Si bien, es cierto, que para que las familias puedan experimentar c $\mathrm{v}$ deben tener antes satisfechas sus necesidades básicas y, de este modo, disfrutar de una vida familiar conjunta que les permita plantearse metas significativas (Turnbull, Summers, Poston y Beegle, 2000). 
Lo que se plantea desde el modelo de CVF es contribuir al empoderamiento de la familia para potenciar su protagonismo, su capacidad de elección y su función de apoyo. La CVF no es algo estático, sino que evoluciona con el tiempo, al cambiar la edad de sus integrantes, incluida la PCD, y las situaciones que afrontan en su vida. Por ello, Giné et al. (2010) reflexionan sobre la inexistencia de una CVF estándar, pues se trata de un concepto que varía según cada familia y depende de la experiencia de cada una de las personas que la conforman.

\section{Principal modelo explicativo de calidad de vida familiar}

El principal modelo aplicado de cVF es el que desarrolló el Beach Center de la Universidad de Kansas en Estados Unidos, que se configura en torno a cinco dimensiones (tabla 1). A su vez, incluye varios indicadores (Hoffman et al., 2006; Poston et al. 2003; Summers et al. 2005, 2007; Turnbull, 2003; Turnbull et al., 2000, 2007). Este modelo tuvo como insumo las demandas de las familias con el fin de que se examinara con precisión su situación, sus fortalezas y necesidades. Por ello, se centra en la colaboración entre familias y profesionales para mejorar la CVF y la de la PcD, como integrante del sistema familiar.

Tabla 1. Modelo de calidad de vida familiar del Beach Center on Disabilities.

\begin{tabular}{|c|c|}
\hline Dimensión & Indicadores \\
\hline \multirow{7}{*}{ Interacción familiar } & Los miembros de mi familia: \\
\hline & - Disfrutan el tiempo juntos \\
\hline & - Se expresan abiertamente unos con otros \\
\hline & - Resuelven los problemas unidos \\
\hline & - Se apoyan unos a otros para alcanzar objetivos \\
\hline & - Se demuestran cariño e interés mutuo \\
\hline & - Hacen frente a los altibajos de la vida \\
\hline \multirow{4}{*}{ Rol parental } & Los miembros de la familia: \\
\hline & $\begin{array}{l}\text { - Ayudan a la persona con discapacidad a: 1) ser independiente; 2) llevar a cabo } \\
\text { sus tareas y actividades; 3) llevarse bien con los demás; y 4) tomar decisiones } \\
\text { adecuadas }\end{array}$ \\
\hline & $\begin{array}{l}\text { - Conocen a otras personas que forman parte de la vida del integrante de la } \\
\text { familia con discapacidad, como amigos o amigas, profesores o profesoras, } \\
\text { entre otros }\end{array}$ \\
\hline & $\begin{array}{l}\text { - Tienen tiempo para ocuparse de las necesidades de la persona con discapa- } \\
\text { cidad }\end{array}$ \\
\hline
\end{tabular}


(viene)

\begin{tabular}{|c|c|}
\hline Dimensión & Indicadores \\
\hline \multirow{5}{*}{ Bienestar emocional } & La familia: \\
\hline & - Cuenta con el apoyo necesario para aliviar el estrés \\
\hline & - Tiene amigos o amigas u otras personas que les brindan su apoyo \\
\hline & - Dispone de algún tiempo para ellos \\
\hline & $\begin{array}{l}\text { - Cuenta con ayuda externa para atender a las necesidades especiales de todos } \\
\text { los miembros de la familia }\end{array}$ \\
\hline \multirow{6}{*}{ Bienestar físico y material } & La familia: \\
\hline & - Cuenta con medios de transporte \\
\hline & - Recibe asistencia médica cuando la necesita \\
\hline & - Puede hacerse cargo de los gastos \\
\hline & - Se siente segura en casa, en el trabajo y en el barrio \\
\hline & - Se siente informada \\
\hline \multirow{5}{*}{$\begin{array}{l}\text { Apoyos relacionados con } \\
\text { la persona con discapa- } \\
\text { cidad }\end{array}$} & La persona con discapacidad tiene apoyo para: \\
\hline & - Progresar en el centro o el trabajo \\
\hline & - Progresar en el hogar \\
\hline & - Hacer amigos o amigas \\
\hline & $\begin{array}{l}\text { - La familia tiene buenas relaciones con los proveedores de servicios que traba- } \\
\text { jan con la persona con discapacidad }\end{array}$ \\
\hline
\end{tabular}

Fuente: adaptada de Summers et al. (2005).

Las dimensiones e indicadores del modelo, basado en el análisis de las opiniones de familiares de PcD, aluden a características específicas del bienestar de los integrantes de la familia, a la calidad de sus interacciones y a la relación con su entorno, particularmente, con los recursos con los que cuenta socialmente.

El desarrollo del modelo de cv centrado en la familia implicó cambios importantes, entre los que se destacan el abandono de la perspectiva patológica en la cual permanecían ideas como las de 'arreglar' a la PCD y a la madre para que lograran el mayor ajuste a los recursos (Rodríguez, 2011). También se reformuló el discurso saturado de connotaciones negativas. Por ejemplo, el término depresión o estrés pasó a entenderse como bienestar o adaptación/funcionamiento familiar (Turnbull et al., 2007) y otras transformaciones (figura 1). 
Miguel Ángel Verdugo Alonso, Leonor Córdoba Andrade, Alba Rodríguez Aguilella 9

Enfoque individual

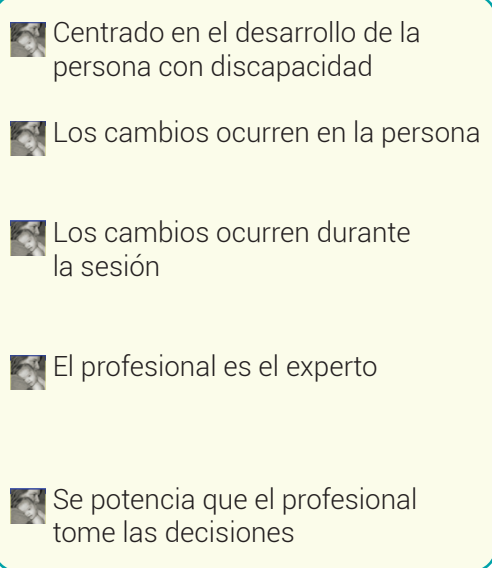

Enfoque sistémico familiar

Centrado en el desarrollo de la persona y el bienestar de la familia

Los cambios ocurren en el bienestar familiar

Los cambios ocurren cuando las rutinas familiares se llevan a cabo de forma natural

Todos los miembros del equipo incluyendo a todos los miembros de la familia, son los expertos

Se empodera a todos los miembros de la familia para tomar decisiones

Figura 1. Modelo de empoderamiento familiar.

Fuente: adaptada de Turnbull (2003).

Desde esta perspectiva, el Modelo de Empoderamiento Familiar (Turnbull, 2003) aboga por la identificación de las necesidades percibidas por las familias y pone a su disposición los servicios de apoyos, con la pretensión de que puedan convertirse en facilitadores del bienestar de la PCD, con los menores riesgos para su salud y sus proyectos de vida (Aguado y Alcedo, 2005). El reto añadido que aporta esta nueva reconsideración del rol de las familias es lograr un mayor ajuste e implicación a nivel familiar, ya que no basta con adaptar los servicios a las familias. El principio al que se ciñe esto, plantea que la CVF es el resultado que debe conseguirse a través de la capacitación de la propia familia, es decir, identificando sus necesidades y dotándola de los recursos necesarios para que puedan establecer sus prioridades y elaborar su propio plan para alcanzarlas, más allá de la adaptación simple de los recursos a sus demandas (Rodríguez, 2011). Esto significa empoderar a las familias para tomar sus propias decisiones en torno a su vida (Córdoba, Verdugo y Gómez, 2008).

\section{La evaluación de la calidad de vida familiar}

A partir de este modelo de CVF, que el Beach Center inició en 1988, en 1997 se estructuró un proyecto internacional desarrollado en Australia, Canadá e Israel con el objetivo de avanzar en la evaluación de la cv de las familias de PCD intelectual -DI(I. Brown et al., 2003; R. Brown et al., 2006; Isaacs et al., 2007; Werner et al., 2009). 
A partir de este trabajo, se han realizado estudios en diferentes contextos, que fueron reseñados en una primera etapa, por Samuel, Rillota y Brown (2012). Estos autores, específicamente, informan sobre los avances que han ocurrido en torno a cVF y DI. Así mismo, evidencian los logros más significativos en cuanto a la evaluación de CVF mediante la contrastación entre el proyecto de Calidad de Vida Familiar (FQOL, por su sigla en inglés) y la iniciativa de CVF del Beach Center.

En la misma dirección, Fernández et al. (2015) escriben sobre los modelos e instrumentos más habituales de evaluación e intervención con familias desde la CV, en el que llaman la atención sobre la inexistencia de consenso en lo que concierne a un modelo único de medición de la CVF, ni tampoco en relación con las dimensiones. Quizá esto, ha incidido en el desarrollo - un tanto lento- de la comprensión y evaluación de la CVF.

No obstante, en el ámbito internacional, la evolución del constructo de CVF y la investigación inicial sobre el mismo, se debe en gran medida al trabajo del Beach Center, que posteriormente ha sido reforzado con los aportes de R. Brown (2008); I. Brown et al. (2003) e I. Brown (2010). Las publicaciones han estado centradas en la construcción de un instrumento de evaluación con propiedades adecuadas de fiabilidad y validez y, a su vez, su consolidación ha permitido avanzar en el desarrollo del constructo. Su utilidad se ha evidenciado en la práctica profesional y de los servicios de atención a la discapacidad, con buenos resultados que se han adoptado para canalizar las inquietudes de profesionales y organizaciones interesadas por trabajar con esa finalidad (Gómez et al., 2010).

Por otra parte, la Escala de Calidad de Vida Familiar (ECVF) del Beach Center (Hoffman et al., 2006) evolucionó desde las diez dimensiones iniciales propuestas por Park et al. (2003) hasta cinco factores vigentes, concretados por Summers et al. (2005). Y, posteriormente, el trabajo se ha centrado en: ayudar a las familias a disponer de la información necesaria para ser capaces de tomar las decisiones que más beneficien a su unidad (Turnbull et al., 2008; Zuna et al., 2009); la obtención de una propuesta del modelo teórico que aúne los planteamientos formulados en investigaciones previas (Zuna et al., 2009); la mejora (Balcells, Giné, Guàrdia y Summers, 2016), adaptación del instrumento de evaluación (Zuna y Kang, 2010; Zuna et al., 2009), su uso en otros contextos (Mckechanie, Moffat, Johnstone y Fletcher-Watson, 2017) y la construcción y calibración de un instrumento orientado a la evaluación de necesidades de la familia, conocido como Cuestionario de Necesidades Familiares, construido por un grupo de investigadores de Estados Unidos, China y España (Baqués, 2016; Chiu, Turnbull y Summers, 2013). Esto con el propósito de contar con un instrumento de evaluación que permita conocer de manera particular y real las necesidades 
familiares de personas con DI y cómo estás repercuten en su CV. Dicho cuestionario ha sido validado en algunos países de América Latina como Brasil (Bitencourt, 2018; Bitencourt, Grácia y Beltrán, 2015), Costa Rica (Cordero, Giné y Balcess, 2019) y Colombia (Aya-Gómez, Ovalle, Cabrera-García, Muñoz y Duque, 2016).

Por otra parte, la encuesta "International Family Quality of Life Survey" desarrollada por Brown et al. (2006) se ha utilizado en varios países (Ajuwon y Brown, 2012; Rillotta, Kirby, Shearer y Nettelbeck, 2012; Viana et al., 2017), cuya estructura multidimensional de la CVF, en este caso, a partir de nueve dimensiones (Isaacs et al., 2007), está en concordancia con la línea de investigación centrada en realizar un análisis transcultural de los resultados obtenidos tras su aplicación en los diferentes países que colaboraron con el proyecto (Hu, Wang y Fei, 2012; I. Brown, 2010; R. Brown, 2008; Petrowski et al., 2008; Werner et al., 2009).

\section{Algunos desarrollos destacables en España y América Latina}

En España y América Latina la investigación se focalizó en la adaptación de la ECVF del Beach Center (Córdoba, Verdugo y Gómez, 2006; Sainz, Verdugo y Delgado, 2006; Verdugo, Córdoba y Gómez, 2005, 2006; Verdugo, Rodríguez y Sainz, 2012). Posteriormente, se utilizó la escala en un buen número de investigaciones que han permitido analizar la CVF en diversos contextos y grupos poblacionales (Aya y Córdoba, 2013; Barrón, Garza, y Vásquez, 2015; Bello et al., 2017; Córdoba, Mora, Bedoya y Verdugo, 2007; Córdoba et al., 2008; Lumani y Córdoba, 2014), así como, relacionarla con otros constructos (Muñoz, Poblete y Jiménez, 2012; Palma, Zapata, Satizabal y Roa, 2016; Verdugo et al., 2009).

En España, un campo destacado de estudio de la CVF, han sido las familias con hijos con DI mayores de 45 años, por tanto, en proceso de envejecimiento tanto los padres como los hijos. En el trabajo de Verdugo, Sánchez y Rodríguez (2009), se ha denominado a esta situación la "doble dependencia". Al respecto, la investigación desarrollada mediante la ECVF y grupos de discusión, se centró en las necesidades percibidas y la CVF a fin de facilitar la planificación de recursos y políticas sociales orientadas a estas familias de PcD (Rodríguez, Verdugo y Sánchez, 2008).

Los resultados de Verdugo, Arias e Ibañez (2007) y Verdugo, Sánchez y Rodríguez (2009) reflejan que interacción familiar, salud y seguridad, y rol parental influyen positivamente sobre la CVF. Sin embargo, las relacionadas con los apoyos, tanto para la familia como para la $\mathrm{PcD}$, revelan un mayor desequilibrio que afecta 
el bienestar percibido, destacándose la insatisfacción con los apoyos recibidos, y la demanda de información, formación y ayuda psicológica. También, se subraya la necesidad de contar con alternativas de ocio y de que los hijos con discapacidad, tengan amigos.

Otro aspecto reseñable de la investigación de Verdugo et al. (2009), es la confirmación del rol de las madres como principales cuidadoras de sus hijos que, cuando tienen una edad avanzada, ven mermadas su salud física y psicológica. A esto se suma el temor por el futuro del hijo con discapacidad -dónde, cómo y con quién vivirán- y la reducción de las redes sociales de apoyo a medida que este integrante va envejeciendo, por lo que la comunicación abierta entre los miembros de la familia y al apoyo prestado entre unos y otros se valora positivamente, además de las relaciones entre los progenitores y los proveedores de servicios.

En España también resaltan los estudios sobre CVF y discapacidad en edades tempranas (Giné, Gràcia, Vilaseca y Balcells, 2009, 2010; Gràcia y Vilaseca, 2008) y el desarrollo de cuestionarios originales de evaluación de la CVF (Balcells, Giné, Guardia y Summers, 2011; Giné et al., 2013) que han tenido amplia difusión en las organizaciones de apoyo a personas con DI.

En América Latina existe otra escala reseñable, aunque de menor uso, para medir la CVF, que fue desarrollada en Argentina por Aznar y Castañón (2005). No obstante, un buen número de estudios se han adelantado a partir de la escala diseñada por el Beach Center. En Colombia, se llevó a cabo un estudio para evaluar la CV de las familias de niños y adolescentes con diferentes discapacidades- (Córdoba et al., 2008). Se encontró que los indicadores en los que las familias informaron sentirse más insatisfechos fueron los relacionados con los apoyos por parte del gobierno y de entidades locales para el acceso a los servicios para el niño o el adolescente con discapacidad. Mientras que las dimensiones orientadas directamente a la familia como interacción familiar, rol parental y salud y seguridad de la familia, tendían a ser positivamente valoradas.

En la misma dirección, otras investigaciones (Aya y Córdoba, 2013; Córdoba et al., 2014) se centraron en el uso del mapa de CVF diseñado por el equipo de investigación del Beach Center, que se adaptó para Colombia (Córdoba et al., 2008; Córdoba et al., 2011) y Argentina (Lumani y Córdoba, 2014). Es un instrumento que sintetiza la información procedente de la evaluación de CVF y favorece la planificación y el seguimiento de la atención (Córdoba et al. 2014; Ortiz, Ariza y Pachajoa, 2018). 


\section{Acuerdos de cooperación entre profesionales y familias en la atención desde calidad de vida}

Finalmente, conviene llamar la atención sobre las estrategias que potencian y consolidan la atención a las familias de PCD desde un enfoque centrado en la familia. Una de ellas es la cooperación entre profesionales y familias (Turnbull et al., 2009). Turnbull et al. (2011) definen el trabajo cooperativo como un acuerdo para generar resultados positivos en la vida de las PcD en función de unos objetivos comunes a partir de los recursos con que se cuenta y las experiencias acumuladas (Córdoba et al., 2008; Córdoba y Verdugo, 2015). A partir de esta estrategia, se busca generar una respuesta más eficaz y contundente, que beneficie directamente a la PcD y, a su vez, se alcancen logros que respondan oportunamente a necesidades y expectativas realistas (Córdoba et al., 2008), contribuyendo plenamente a la CV tanto de la familia como de la PcD. De este modo, estos acuerdos interpelan a los profesionales a poner al servicio de la familia, el conocimiento más actualizado y completo basado en la evidencia, que les permita tomar las decisiones más acertadas sobre los aspectos y objetivos más significativos e importantes de sus vidas (Rivard et al., 2017), a partir de la premisa: "la familia se constituye en el centro de la atención y el objetivo de dicha atención es resaltar las fortalezas de la familia para responder a sus necesidades" (Córdoba y Verdugo, 2015, p. 6).

\section{Conclusiones}

En el ámbito de la discapacidad, y desde una perspectiva ecológica-contextual, es evidente la relación entre $C V$ individual y la CVF, ya que lo que ocurre en la vida de la PCD, al ser un integrante de la familia, necesariamente va a influir en la vida de los demás miembros de su grupo familiar, y a la vez, cada una de las circunstancias vitales por las que atraviesan los padres, los hermanos y demás personas que conforman este microsistema, tienen un impacto en la vida de la PcD.

Sin duda, los esfuerzos que se han realizado en las dos últimas décadas por parte de algunos equipos de investigación, entre los que se destaca el Beach Center on Disabilities de la Universidad de Kansas en Estados Unidos y el de Brown y Brown en Canadá, han contribuido de manera relevante a la comprensión, evaluación y atención a las familias de PCD desde el modelo de CVF, que se operativiza en cinco dimensiones: interacción familiar, rol de padres o rol parental, salud y seguridad de 
la familia, recursos generales y, apoyos para la persona con discapacidad. Alrededor de estas dimensiones se construyó la Escala de CVF, adaptada en España y algunos países de América Latina como Colombia, Argentina, Chile y México, con la pretensión de aportar a una práctica profesional y organizacional basada en la evidencia.

Los estudios realizados hasta el momento en torno a cV en familias de PcD, en contextos diversos y con grupos poblacionales diferentes, han demostrado la utilidad práctica que representan la Escala y el Mapa de CVF, sumado al desarrollo de estrategias de indagación sobre las fortalezas y necesidades que repercuten en la cV de estas familias (por ejemplo, el Cuestionario de Necesidades Familiares), como insumos para un seguimiento de los procesos de atención basados en el empoderamiento de las familias, que traen como consecuencia una serie de transformaciones desde la propia concepción de familia así como, en su funcionamiento; una actitud de humildad y practicidad por parte de los profesionales, quienes asumen una relación más colaborativa y menos de "intervención" a partir del reconocimiento de que ellos no son los expertos, sino integrantes del equipo, es decir, familia y profesionales, en un marco de respeto hacia las rutinas y características individuales de cada sistema familiar.

Por último, si bien se ha avanzado en esta tarea, es indispensable que continuemos trabajando en esta dirección, para ser coherentes con la Convención de los Derechos de las Personas con Discapacidad, promulgado por las Naciones Unidas en 2006 y que, de una u otra manera y a diferentes ritmos, se viene adoptando tanto en España, como en América Latina. Por lo tanto, la preocupación, el esfuerzo y las tareas tanto de los padres de PcD como de los profesionales de apoyos, se fundamentan en el desarrollo personal y la inclusión social de las PcD, desde la cooperación, el respeto y el reconocimiento de las características particulares de este colectivo.

\section{Referencias}

Aguado, A. L. y Alcedo, M. A. (2005). Una visión psicosocial del envejecimiento de las personas con lesión medular. Intervención Psicosocial, 14(1), 51-63.

Ajuwon, P. M. y Brown, I. (2012). Family Quality of Life in Nigeria. Journal of Intellectual Disability Research, 56(1), 61-70. Recuperado de: https://www.ncbi.nlm.nih.gov/pubmed/22049915.

Aya, V. y Córdoba, L. (2013). Asumiendo juntos los retos: calidad de vida en familias de jóvenes con discapacidad intelectual. Revista Facultad de Medicina, 61(2), 155-166. 
Aznar, A. S. y Castañon, D. G. (2005). Quality of Life from the Point of View of Latin American Families: A Participative Research Study. Journal of Intellectual Disability Research, 49(10), 784-788. https://doi.org/10.1111/j.1365-2788.2005.00752.x

Aya-Gómez, V., Ovalle, A., Cabrera-Garcia, V., Muñoz, D. y Duque, C.A. (2016). Validation of the Family Needs Assessment Scale for Colombian Families with Children with Intellectual Disability. International Journal on Disability and Human Development, 16(3), 311-319. https://doi. org/10.1515/ijdhd-2016-0033

Balcells, A., Giné, C., Guàrdia, J. y Summers, J. A. (2011). Family Quality of Life: Adaptation to Spanish Population of Several Family Support Questionnaires. Journal of Intellectual Disability Research, 55(12), 1151-1163. https://doi.org/10.1111/j.1365-2788.2010.01350.x

Balcells, A., Giné, C., Guàrdia, J. y Summers, J. A. (2016). Proposal of indexes to evaluate Family Quality of Life, Partnership and Family Support Needs. Revista Iberoamericana de Psicología y Salud, 7(1), 31-40. http://dx.doi.org/10.1016/j.rips.2015.10.005

Baqués, N. (2016). La valoración de las necesidades de apoyo de las familias con un hijo/a con discapacidad intelectual. Validación de una escala (Tesis Doctoral). Barcelona: Universitat de Barcelona.

Barrón, F., Garza, T. y Vásquez, D. (2015). Mejoría en la calidad de vida en familias con hijos con parálisis cerebral con intervención psicoeducativa. Revista Mexicana de Medicina Física y Rehabilitación, 27(1), 12-16. Recuperado de: http://www.medigraphic.com/pdfs/fisica/mf2015/mf151c.pdf

Beach Center (2001). Family Quality of life Survey. Kansas: University of Kansas-Beach Center of Disability.

Bello, N., Rivadeneira, J., Concha, M., Soto, A. y Díaz, X. (2017). Escala de CVF: validación y análisis en población chilena. Universitas Psychologica, 16(4). https://doi.org/10.11144/Javeriana. upsy16-4.ecvf

Bitencourt, D. S. (2018). Evaluación de las necesidades de apoyo de las familias de personas con Discapacidad Intelectual y/o del Desarrollo en Brasil (Tesis Doctoral). Barcelona: Universitat de Barcelona.

Bitencourt, D. S., Gràcia, M. y Beltrán, F. S. (2015). Evaluación de las necesidades de las familias con adultos con discapacidad intelectual en Brasil. Ponencia presentada en las IX Jornadas Científicas Internacionales de Investigación sobre Discapacidad. INICO, Salamanca: Universidad de Salamanca. 
Bronfenbrenner, U. (1974). Developmental Research, Public Policy and the Ecology of Childhood. Child Development, 32, 513-531.

Bronfenbrenner, U. (2005). Making Human Beings Human. Bioecological Perspectives on Human Development. Oaks: Sage.

Brown, I. (2010). Family Quality of Life: Comparison Among 13 Countries. Journal of Applied Research in Intellectual Disabilities, 23(5), 502. https://doi.org/10.1111/j.1468-3148.2010. 00590.x

Brown, I., Anand, S., Fung, W. L. A., Isaacs, B. y Baum, N. (2003). Family Quality of Life: Canadian Results from an International Study. Journal of Developmental and Physical Disabilities, 15(9), 207-215. https://doi.org/10.1023/A:1024931022773

Brown, R. (2008). Family Quality of Life: Reappraisal of Challenges and Supports Where There is a Child with Complex Disability. Inspire, 2 (2), 4-8.

Brown, R., Hong, K., Shearer, J., Wang, M. y Wang, S. (2010). Family Quality of Life in Several Countries: Results and Discussion of Satisfaction in Families Where There is a Child with a Disability. En R. Kober (Ed.), Enhancing the quality of life of people with intellectual disabilities (pp. 377-398). Nueva York: Springer.

Brown, R., MacAdam-Crisp, J., Wang, M. y Larocci, G. (2006). Family Quality of Life When There is a Child with Developmental Disability. Journal of Policy and Practice in Intellectual Disabilities, 3, 238-245. https://doi.org/10.1111/j.1741-1130.2006.00085.x

Chiu, C., Kyzar, K., Zuna, N., Turnbull, A., Summers, J. A. y Aya, V. L. (2013). Family Quality of Life. In M. L. Wehmeyer (Ed.), The Oxford Handbook of Positive Psychology and Disability (pp. 365-392). Nueva York: Oxford University Press.

Chiu, C., Turnbull, A. P. y Summers, J. A. (2013). What Families Need: Validation of the Family Needs Assessment for Taiwanese Families of Children with Intellectual Disability and Developmental Delay. Research and Practice for Persons with Severe Disabilities, 38 (4), 247-258.

Cordero, B., Giné, C. y Balcells, A. (2019). Necesidades y calidad de vida de familias de personas en edades tempranas con discapacidad en Costa Rica. Adaptación y validación de una escala. Revista Terapéutica, 13(1), 6-21.

Córdoba, L. (2017). Calidad de vida, un modelo clave en la atención a personas con discapacidad intelectual desde la perspectiva ecológica. Revista Electrónica de Educación Especial y Familia, 8 (2), 6-13. 
Córdoba, L. y Verdugo, M. A. (2015). Acuerdos de cooperación entre profesionales y familias. Ponencia presentada en las IX Jornadas Científicas Internacionales de Investigación sobre Personas con Discapacidad. INICO, Salamanca: Universidad de Salamanca.

Córdoba, L., Aya, V., Ibáñez, A., Monsalve, A. y Gómez, M. C. (2011). Herramientas de evaluación para medir el impacto de programas de transición a la vida adulta dirigidos a jóvenes con discapacidad intelectual. Documentos de Investigación. Escuela de Medicina y Ciencias de la Salud, 13, 7-14.

Córdoba, L., Gómez, J. y Verdugo, M. A. (2008). CVF en personas con discapacidad: un análisis comparativo. Universitas Psychologica, 7(2), 369-383.

Córdoba, L., González, M., Obando, D. P. y Colacouglou, C. (2013). Current Trends in Family Structures in Four Colombian Cities. Population Review, 52, 119-134.

Córdoba, L., Mora, A., Bedoya, A. M. y Verdugo, M. A. (2007). Familias de adultos con discapacidad intelectual en Cali, Colombia, desde el modelo de calidad de vida. Psykhe, 16(2), 29-42.

Córdoba, L., Verdugo, M. A. y Gómez, J. (2006). Adaptación de la Escala de CVF en Cali (Colombia). En: M. A. Verdugo (Dir.). Cómo mejorar la calidad de vida de las personas con discapacidad. Instrumentos y estrategias de evaluación. (pp. 273-298). Salamanca: Amarú.

Córdoba, L., Verdugo, M. A., Aya-Gómez, V. y Lumani, N. (2014). Investigación y atención psicosocial de familias de personas con discapacidad: un enfoque práctico por medio del mapa de CVF. siglo Cero, 45(4), 48-62.

Cuevas, M. M., Salcedo, R. y Díaz, F. (2019). Discapacidad y familia en contextos multiculturales. Un estudio sobre la realidad en la ciudad autónoma de Ceuta. Revista Pedagogía Social, (33), 111-126.

Fernández, A., Montero, D., Martínez, N., Orcasitas, J. R. y Villaescusa, M. (2015). CVF: marco de referencia, evaluación e intervención. Siglo Cero, 46(2), 7-29. http://dx.doi.org/10.14201/ scero2015462729

García, P., McWilliams, R. A., Martínez-Rico, G. y Morales-Murrillo, C. (2018). Child, Family, and Early Intervention Characteristics Related to Family Quality of Life in Spain. Journal of Early Intervention, 1-18. https://doi.org/10.1177/1053815118803772

Giné, C., Gràcia, M., Vilaseca, R. y Balcells, A. (2009). Trabajar con las familias en Atención Temprana. Revista Interuniversitaria de Formación del Profesorado, 65 (23, 2), 95-113. 
Giné, C., Gràcia, M., Vilaseca, R. y Balcells, A. (2010). Quality of Life of the Families of People with Intellectual Disability in Spain. En R. Kober (Eds.). Enhancing the Quality of life of People with Intellectual Disabilities. From Theory to Practice (pp. 349-362). Nueva York: Springer.

Giné, C., Grácia, M., Vilaseca, R., Salvador Beltrán, F., Balcells, A., Dalmau, M., et al. (2015). Family Quality of Life for People With Intellectual Disabilities in Catalonia. Journal of Policy and Practice in Intellectual Disabilities, 12(4), 244-254. https://doi.org/10.1111/.jppi.12134

Giné, C., Vilaseca, R., Gràcia, M., Simón, C., Dalmau, M., Salvador, F. y Cagigal, V. (2013). Las Escalas de CVF (CDVF-E). Apoyo y orientación para la intervención. Siglo Cero, 44(247), 21-36.

Gómez, L. E., Verdugo, M. Á. y Arias, B. (2010). Calidad de vida individual: avances en su conceptualización y retos emergentes en el ámbito de la discapacidad. Psicología conductual, 18, 453-472.

Gràcia, M. y Vilaseca, R. (2008). Cómo mejorar la calidad de vida de las familias de niños con discapacidad intelectual: algunas propuestas. Siglo Cero, 39(226), 44-62.

Guillén, V., Verdugo, M.A., Arias B. y Vicente, E. (2015). Desarrollo de una escala de evaluación de necesidades de apoyo de niños y adolescentes con discapacidad intelectual. Anales de Psicología, 31(1), 137-144. https://doi.org/10.6018/analesps.31.1.166491

Hart, K. E.y Sasso, T. (2011). Mapping the contours of contemporary positive psychology. Canadian Psychology, 52, 82-92. Recuperado de: http://psycnet.apa.org/journals/cap/52/2/82.pdf

Hoffman, L., Marquis, J., Poston, D., Summers, J. A. y Turnbull, A. (2006). Assessing Family Outcomes: Psychometric Evaluation of the Beach Center Family Quality of Life Scale. Journal of Marriage and Family, 68(4), 1069-1083. https://doi.org/10.1111/j.1741-3737.2006.00314.x

Hu,X., Wang, M., y Fei,X. (2012). Family Quality of Life ofChinese Families of Children with Intellectual Disabilities. Journal of Intellectual Disability Research, 56(1), 30-44. Recuperado de: https:// www.ncbi.nlm.nih.gov/pumed/21366752.

Isaacs, B. J., Brown, I. Brown, R., Baum, N., Myerscough, T., Neikrug, N., et al. (2007). The International Family Quality of Life Project: Goals and Descriptions of a Survey Tool. Journal of Policy and Practice in Intellectual Disabilities, 4 (3), 177-185. https://doi. org/10.1111/j.1741-1130.2007.00116.x 
Kovac, A. y Memisevic, H. (2017). Quality of Life of Parents of Children with Intellectual Disabilities in Croatia. Journal of Educational and Social Research, 7 (2), 43-48. https://doi.org/10.5901/ jesr.2017.v7n2p43

Luckasson, R. y Schalock, R. L. (2013). Defining and Applying a Functionality Approach to Intellectual Disability. Journal of Intellectual Disability Research, 57, 657-668. https://doi. org/10.1111/j.1365-2788.2012.01575.x

Luckasson, R., Borthwick-Duffy, S., Buntix, W.H.E., Coutler, D.L., Craig, E.M., Reeve, A. et al. (2002). Mental Retardation. Definition, Classification and System of Supports (10th Ed.). Washington: American Association on Mental Retardation. [Traducción al castellano de M.A. Verdugo y C. Jenaro (2004). Madrid: Alianza Editorial].

Lumani, N. y Córdoba, L. (2014). Satisfacción con la CVF y con los servicios en familias de niños y adolescentes con discapacidad. Siglo Cero, 45(1), 41-55.

Martínez, N., Fernández, A., Orcasitas, J. R. Montero, D. y Villaescuesa, M. (2016). Diseño de un programa de apoyo a la calidad de vida de familias con jóvenes y adultos con discapacidad intelectual. Siglo cero, 47(3), 47-67. http://dx.doi.org/10.14201/scero20164734767

Mckechanie, A.G., Moffat, V.J., Johnstone, E.C. y Fletcher-Watson, S. (2017). Links between Autism Spectrum Disorder Diagnostic Status and Family Quality of Life. Children, 4(4), 1-11. https:// doi.org/10.3390/children4040023

Mora, A., Córdoba, L., Bedoya, A. M. y Verdugo, M. A. (2007). Características de la calidad de Vida en familias con un adulto con discapacidad intelectual (DI)/RM en la ciudad de Cali, Colombia. Diversitas, 3(1), 37-54.

Muñoz, G. (2019). Resignificación del trayecto vital de mujeres-madres de hijos e hijas con discapacidad: de la experiencia a la construcción de saberes (Tesis de Maestría). Bogotá: Universidad Pedagógica Nacional.

Muñoz, Y., Poblete, Y. y Jiménez, A. (2012). CVF y bienestar subjetivo en jóvenes con discapacidad intelectual de un establecimiento con educación especial y laboral de la Ciudad de Talca. In terdisciplinaria, 29 (2), 207-221.

Navas, P., Gómez, L. E., Verdugo, M. A. y Schalock, R. L. (2012). Derechos de las personas con discapacidad intelectual: omplicaciones de la Convención de las Naciones Unidas, Siglo Cero, 43(3), 7-28. 
Organización Mundial de la Salud (OMS). (2001). Clasificación internacional del funcionamiento de la discapacidad y de la salud: CIF. Madrid: Ministerio de Trabajo y Asuntos Sociales.

Organización de las Naciones Unidas (ONU) (2006). Convention on the Rights of Persons with Disabilities. Recuperado de: http://www.un.org/disabilities/convention/conventionfull. shtml.

Ortiz, D. M., Ariza, Y. y Pachajoa, H. (2018). Calidad de vida de familias de niños y adolescentes con discapacidad asociada a defectos congénitos. Universitas Psychologica, 17(1). https://doi. org/10.11144/Javeriana.upsy17-1.cvfn

Palma, D., Zapata, J.S., Satizabal, M. y Roa, P.E. (2016). Participación y Calidad de Vida en familias de personas con discapacidad. Revista Ocupación Humana, 16(1), 19-31.

Park, J., Hoffman, L., Marquis, J. Turnbull, A. P., Poston, D., Mannan, H. et al. (2003). Toward Assessing Family Outcomes of Service Delivery: Validation of a Family Quality of Life Survey. Journal of Intellectual Disability Research, 47(4/5), 367-384.

Petrowski, N.T., Edwards, M., Isaacs, B.J., Baum, N. y Brown, I. (2008). Family quality of life: preliminary analyses from an on-going project. Journal on Developmental Disabilities, 14(2), 111-114.

Poston, D., Turnbull, A., Park, J., Mannan, H., Marquis, J. y Wang, M. (2003). Family Quality of Life: A Qualitative Inquiry. Mental Retardation, 4, 313-328. https://doi.org/10.1352/0047-6765(2003) 41<313:FQOLAQ>2.0.CO;2

Rillotta, F., Kirby, N., Shearer, J. y Nettelbeck, T. (2012). Family Quality of Life of Australian Families with a Member with an Intellectual/Developmental Disability. Journal of Intellectual Disability Research, 56(1), 71-86.

Rivard, M., Mercier, C., Mestari, Z., Terroux, A., Mello, K. y Bégin, J. (2017). Psychometric Properties of the Beach Center Family Quality of Life in French-Speaking Families With a PreschoolAged Child Diagnosed With Autism Spectrum Disorder. American Journal on Intellectual and Developmental Disabilities, 122(5), 439-452. https://doi.org/10.1352/1944-7558-122.5.439

Rodríguez, A., Verdugo, M. A. y Sánchez, M. (2008). CVFy apoyos para los progenitores de personas con discapacidad intelectual en proceso de envejecimiento. Siglo Cero, 39 (3), 19-34.

Rodríguez, A. (2011). Familia, discapacidad intelectual y envejecimiento. Análisis de la CVF desde una perspectiva multidimensional. INICO-Universidad de Salamanca, Tesis doctoral no publicada. 
Sainz, F., Verdugo, M. A. y Delgado, J. (2006). Adaptación de la Escala de CVF al contexto español. En Verdugo, M. A. (Dir.), Cómo mejorar la calidad de vida de las personas con discapacidad (pp. 299-322). Salamanca: Amarú.

Samuel, P.S., Rillota, F. y Brown, I. (2012). The Development of Family Quality of Life Concepts and Measures. Journal of Intellectual Disability Research, 56 (1), 1-16. https://doi. org/10.1111/j.1365-2788.2011.01486.x

Schalock, R. L. y Luckasson, R. (2015). A Systematic Approach to Subgroup Classification in Intellectual Disability. Intellectual and Developmental Disabilities, 53(5), 358-366. https://doi. org/10.1352/1934-9556-53.5.358

Schalock, R. L., Borthwick-Duffy, S. A., Bradley, V. J., Buntinx, W. H. E., Coulter, D. L., Craig, E. M. y Yaeger, M. (2010). Intellectual disability: Diagnosis, classification, and systems of supports (1 $1^{\text {th }}$ ed.). Washington: American Association on Intellectual and Developmental Disabilities.

Schalock, R. L., Verdugo, M. A., Gomez, L. E., y Reinders, H. S. (2016). Moving Us Toward a Theory of Individual Quality of Life. American Journal on Intellectual and Developmental Disabilities, 121(1), 1-12. https://doi.org/10.1352/1944-7558-121.1.1

Schalock, R. y Verdugo, M. A. (2007). El concepto de Calidad de vida en los servicios y apoyos para personas con discapacidad intelectual. Siglo Cero, 38 (4), 21-36.

Schlebusch, L., Dada, S. y Samuels, A. E. (2017). Family Quality of Life of South African Families Raising Children with Autism Spectrum Disorder. Journal of Autism and Developmental Disorders, 47(7), 1966-1977. https://doi.org/10.1007/s10803-017-3102-8

Summers, A., Marquis, J., Mannan, H., Turnbull, A., Fleming, K., Poston, D. et al. (2007). Relationship of perceived adequacy of services, family-professional partnerships, and family quality of life in early childhood service programmes. International Journal of Disability, Development and Education, 54(3), 319-338. https://doi.org/10.1080/10349120701488848

Summers, J. A., Poston, D. J., Turnbull, A. P., Marquis, J., Hoffman, L., Mannan, H. y Wang, M. (2005). Conceptualizing and Measuring Family Quality of Life. Journal of Intellectual Disability Research, 49(10), 777-783. https://doi.org/10.1111/j.1365-2788.2005.00751.x

Thompson, J. R., Wehmeyer, M. L., Hughes, C., Shogren, K. A., Seo, H., Little, T. D. y Schalock, R. (2016). Supports Intensity Scale - Children's Version User's Manual. Washington: American Association on Intellectual and Developmental Disabilities. 
Turnbull, A. (2003). Herramientas para mejorar la calidad de vida de la familia. Documento presentado en las V Jornadas Científicas de Investigación sobre personas con Discapacidad, Salamanca, España.

Turnbull, A., Summers, J., Lee, S. H. y Kyzar, K. (2007). Conceptualization and Measurement of Family Outcomes Associated with Families of Individuals with Intellectual Disabilities. Mental Retardation, 13(4), 346-356. https://doi.org/10.1002/mrdd.20174

Turnbull, H., Summers, J., Poston, D. y Beegle, G. (2000). Enhancing Family Quality of Life Through Partnerships and Core Concepts of Disability Policy. Trabajo presentado en el 11th IASSID World Congress, Seattle, Estados Unidos.

Turnbull, A., Turnbull, R., Erwin, E., Soodak, L. y Shogren, K. (2011). Families, Professionals, and Exceptionality: Positive Outcomes Through Partnerships and Trust. Nueva Jersey: Pearson.

Turnbull, A., Turnbull, R. y Kyzar, K. (2009). Cooperación entre familias y profesionales como fuerza catalizadora para una óptima inclusión: enfoque de los Estados Unidos de América. Revista de Educación, (349), 69-99.

Turnbull, A., Zuna, N. y Gotto, G. (2008). Enhancing Family Quality of Life Through Wisdom-Based Action. Insipire, 2, 14-18.

Verdugo, M. A. (2000). Calidad de vida en las familias con hijos con discapacidad intelectual. En M.A. Verdugo (Ed.), Familias y discapacidad intelectual (pp. 183-198). Madrid: FEAPS/ Caja Madrid.

Verdugo, M. A., Arias, B. elbáñez, A. (2007). Escala de Intensidad de Apoyos (SIS). Manual. Adaptación española [Supports Intensity Scale-SIS Manual. Spanish Version] Madrid: TEA.

Verdugo, M. A., Córdoba, L. y Gómez, J. (2005). Spanish Adaptation and Validation of the Family Quality of Life Survey. Journal of Intellectual Disability Research, 49(10), 794-798. https://doi. org/10.1111/j.1365-2788.2005.00754.x

Verdugo, M. A., Córdoba, L. y Gómez, J (2006). Adaptación y validación al español de la Escala de CVF (ECVF). Siglo Cero, 37(2), 41-46.

Verdugo, M. A., Córdoba, L., Restrepo, A., Cardona, J. y Peña, P. (2009). Psychosocial Factors Affecting Adults with Intellectual Disabilities with Psychiatric Disorders in Cali, Colombia. Journal of Policy and Practice in Intellectual Disabilities, 6 (3), 173-179. https://doi. org/10.1111/j.1741-1130.2009.00223.x 
Verdugo, M. A., Navas, P. A., Gomez, L. E. y Schalock, R. L. (2012). The Concept of Quality of Life and Its Role in Enhancing Human Rights in the Field of Intellectual Disability. Journal of IntellectualDisabilityResearch,56,1036-1045.https://doi.org/10.1111/j.1365-2788.2012.01585.x

Verdugo, M. A., Sánchez, M. C. y Rodríguez, A. (2009). Familias y personas con Discapacidad Intelectual en proceso de envejecimiento: La doble dependencia. Madrid: Síntesis.

Verdugo, M. A., Rodríguez, A. y Sainz, F. (2012). Escala de CVF. Salamanca: Publicaciones INICO, Colección Herramientas Nº 12.

Verdugo, M. A. y Schalock, R. L. (Coords.) (2013). Discapacidad e inclusión. Manual para la docencia. Salamanca: Amarú.

Viana, R.V., De arruda, V., Retto, L., Ramos, C. y Gusmão, D. (2017). Impacto da deficiência intelectual moderada na dinâmica e na qualidade de vida familiar: um estudo clínico-qualitativo. Cadernos de Saúde Pública, 33(11), e00096016. https://doi.org/10.1590/0102-311×00096016

Werner, S., Edwards, M., Baum, N., Brown, I., Brown, R. I. y Isaacs, B. J. (2009). Family Quality of Life Among Families with a Member Who Has an Intellectual Disability: An Exploratory Examination of Key Domains and Dimensions of the Revised FQOL Survey. Journal of Intellectual Disability Research, 53 (6), 501-511. https://doi.org/10.1111/j.1365-2788.2009.01164.x

Wehmeyer, M. L. (Ed.). (2013). The Oxford Handbook of Positive Psychology and Disability. Nueva York: Oxford University Press.

Zuna, N. I. y Kang, S. (2010). Qualitative Examination of Family Quality of Life from the Perspective of Latino Families. Journal of Applied Research in Intellectual Disabilities, 23(5), 504. https:// doi.org/10.1111/j.1468-3148.2010.00590.x

Zuna, N. I., Selig, J. P., Summers, J. A. y Turnbull, A. P. (2009). Confirmatory Factor Analysis of a Family Quality of Life Scale for Families of Kindergarten Children without Disabilities. Journal of Early Intervention, 31(2), 111-125. https://doi.org/10.1177/1053815108330369

Zuna, N. I., Summers, J. A., Turnbull, A. P., Hu, X. y Xu, S. (2009). Theorizing about Family Quality of Life. En R. Kober (Eds.). Enhancing the Quality of Life of People with Intellectual Disability: From Theory to Practice (pp. 241-276). Nueva York: Springer.

Zuna, N. I., Turnbull, A. y Summers, J. A. (2009). Family Quality of Life: Moving from Measurement to Application. Journal of Policy and Practice in Intellectual Disabilities, 6, 25-31. https://doi. org/10.1111/j.1741-1130.2008.00199.x 